\title{
Carcinoma Colon of a Child presenting As Abdominal Pain
}

\author{
MAMTAZ BEGUM ${ }^{1}$, ZOHORA JAMEELA KHAN ${ }^{2}$, MD. KAMRUL HASAN ${ }^{3}$, SABINA KARIM ${ }^{4}$
}

\section{Introduction}

Carcinoma of the colon and rectum is an unusual malignancy in children and it represents about $1.5 \%$ of the total number of malignant solid tumors in pediatric age group. ${ }^{1}$ Other studies showed that less than $1 \%$ of all malignant growths of the large intestine occur under 20 years of age. ${ }^{2,3}$ Colorectal carcinoma is the third most frequent cancer in adult men and the second most common in adult women. ${ }^{4}$ In children it may not be associated with a family history of large bowel cancer. ${ }^{5,6}$ The time interval between the onset of symptoms to diagnosis was about 7.5 months to as long as 2 years. ${ }^{7}$ Gender distribution of colorectal carcinoma in adult is equal where as in children male: female ratio is $2: 1 .^{1,} 8$ The signs and symptoms of colonic carcinoma in children are non specific. Usual symptoms of colorectal cancer in children include changes in bowel habits (constipation, diarrhea, bloating, and pain with bowel movements), an abdominal mass, weight loss, decreased appetite and blood in the stool. When the tumor is suspected, the stool should be checked for blood and the levels of carcinoembryonic antigen (CEA) in the blood should be measured. An examination of the large bowel by

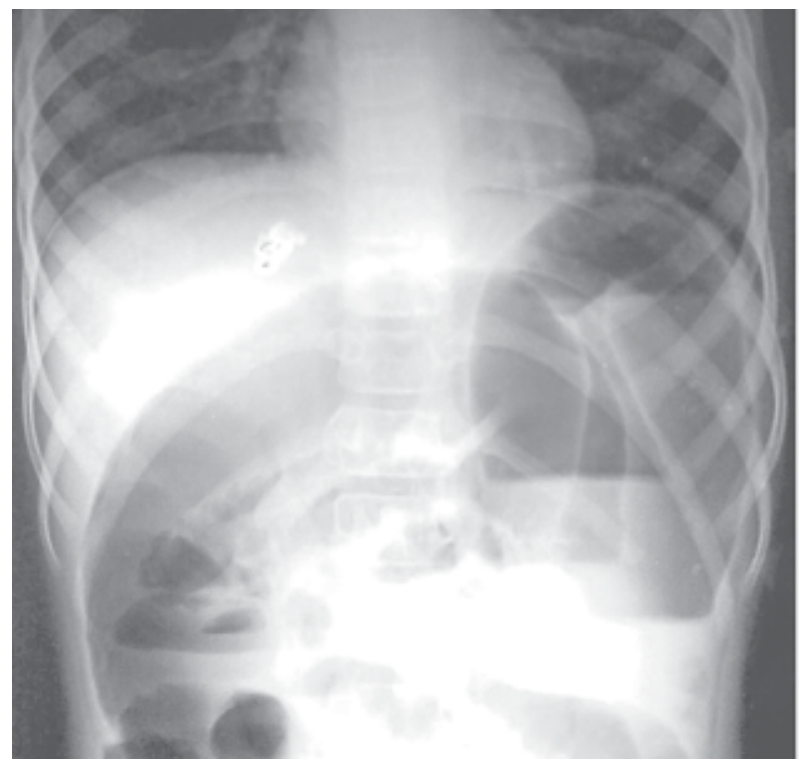

fiber optic colonoscopy or CT colonoscopy should be done. Other radiographic tests like ultasonography of the whole abdomen, a CT scan or MRI of the abdomen also done. Patients are also examined for metastasis using CT scans of the chest and bone scans.

\section{Case Report}

A male child of 11 years and 10 months of age was admitted with the history of operation for acute abdomen. Before that operation this patient had abdominal pain and vomiting off and on for 2 months. Suddenly he developed abdominal distension and constipation for 3 days. At first he was treated by a local doctor but no improvement. Then he was referred in a hospital where enema simplex was given. This causes slight release of abdominal distension and passing of hard stool. He was discharged from the hospital on that day but on the evening again he developed abdominal pain, vomiting and abdominal distension. That time he went to a local doctor who referred him to hospital. There intestinal obstruction was diagnosed by plain X-ray abdomen and also by ultrasonography of abdomen. He had no family history of carcinoma colon or familial polyposis coli. Emergency laparotomy was done there and a growth

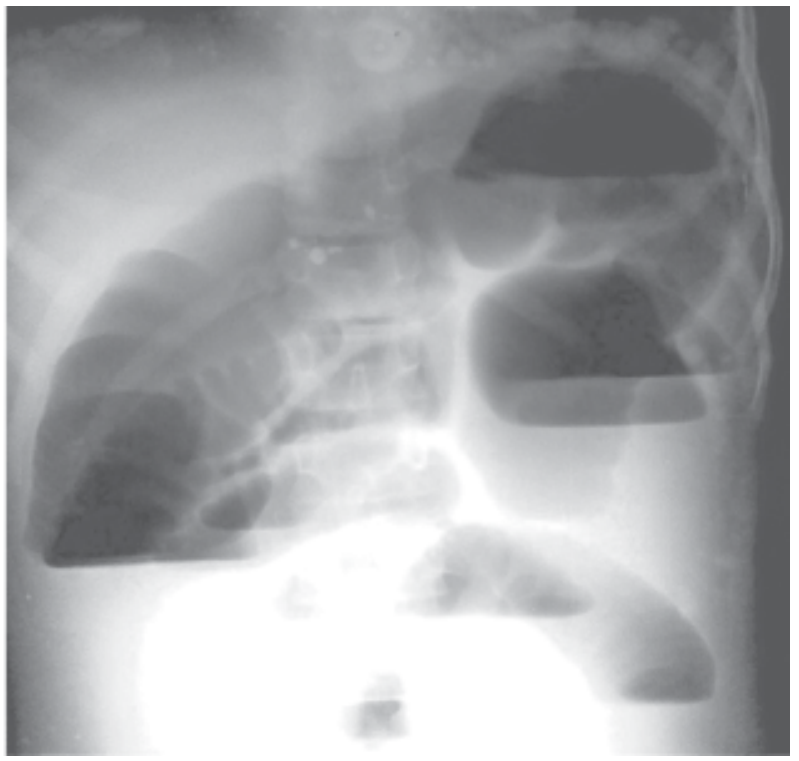

Fig.-1: Plain X-ray abdomen showing features of intestinal obstruction before surgery.

1. Associate professor and head, Department of Paediatric oncology, National Institute of Cancer Research and Hospital (NICRH), Dhaka

2. Assistant Professor, Department of Paediatric Haematology \& Oncology, Dhaka Medical College, Dhaka.

3. Assistant Professor, Department of Haematology, National Institute of Cancer Research and Hospital (NICRH), Dhaka.

4. Registrar, Department of Paediatric oncology, National Institute of Cancer Research and Hospital (NICRH), Dhaka.

Correspondence: Mamtaz Begum 

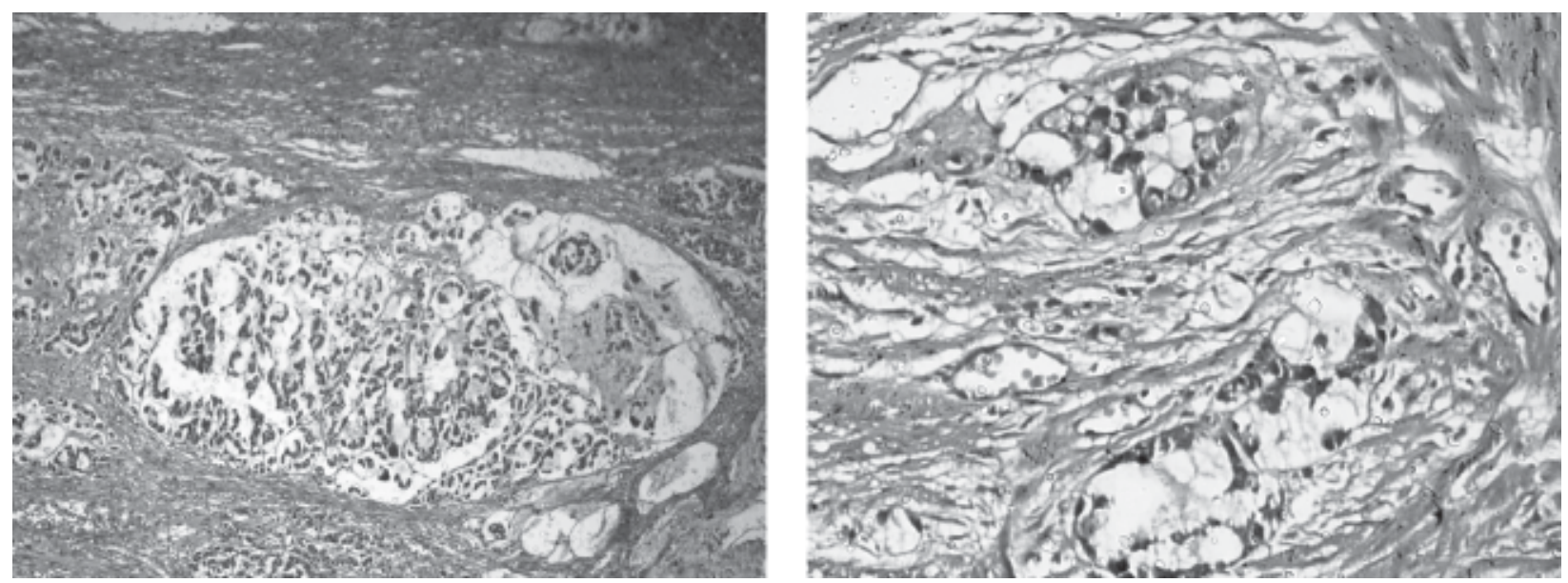

Fig.-2: Histopathological picture of the colonic tissue showing features of moderately differentiated adenocarcinoma.

was found in proximal sigmoid colon. Resection of sigmoid colon with colostomy was done. Histopathology showed adenocarcinoma, moderately differentiated with lymph node metastasis. Anthropometry shows his weight for age is $-3.2 \mathrm{Z}$ score and height for age is -1.6 . Haematolgical parameters were within normal limit. Post operative CEA and LDH were also within normal range. Before giving chemotherapy, assessment of his liver and kidney function tests were done and results were normal. Patient received chemotherapy with cisplatin and 5 FU. After chemotherapy, general condition of the patient was well.

\section{Discussion}

In our country, there is no population based cancer registry. Therefore, it is not possible to find out the incidence of colorectal carcinoma in children in Bangladesh. From January 2010 to December 2012, 751 new pediatric patients of different solid malignancies were attended in the pediatric oncology department of $\mathrm{NICRH}$. Among them, only 10 patients had colorectal carcinoma. In US incidence of colonic carcinoma in children under 20 years of age was estimated to be 1.3 per one million. ${ }^{9}$ One to three colon carcinomas were identified annually among 700 pediatric malignant solid tumors in Japan. ${ }^{10}$ The prevalence of childhood colonic carcinoma in Taiwan was 2 in one million (based on a population of children under 20 years of age of about 7.5 million in Taiwan). ${ }^{11}$

Our case showed non specific symptoms which is consistent with various studies that showed the symptoms of colon cancer in children are non-specific and include chronic persistent abdominal pain, vomiting, constipation/diarrhea, weight loss, occult blood in the stool with anemia, changes in bowel habits, tenesmus, and a palpable abdominal mass. ${ }^{10}$, 12-14 Patients may present with acute intestinal obstruction as seen in our case. Lewis et al found that $63 \%$ of colon cancer patients present with pain, anemia and abdominal distension where as $75 \%$ with rectal bleeding. ${ }^{7}$

Peri-umbilical and epigastric pain may also be present in 10 to $15 \%$ school aged children. ${ }^{15,16}$ These pains are indistinguishable from non organic recurrent abdominal pain that usually misleads and delays the diagnosis of colon carcinoma in children. Such problem occurred in this case also.

Most childhood colon cancer occurred in the late childhood and adolescence such as 9 to 17 years of age and the age of our patient was within this range. Kern et el reported a 9-month-old infant with a mucin secreting adenocarcinoma. ${ }^{5}$

An abdominal ultrasound examination and barium enema studies are necessary diagnostic tools in children suffering from persistent abdominal pain with any warning signs, like weight loss, anemia, and positive abdominal or rectal tenderness. Rectal digital examination, which can detect the signs of rectal tenderness, bleeding, or a palpable mass, may be helpful in evaluation but it is not absolute. The diagnosis is best confirmed by contrast enema and an endoscopic biopsy. ${ }^{15} \mathrm{~A}$ full-course colonoscopic examination is preferred rather than merely sigmoidoscopy. For preoperative staging and postoperative follow-up, Computed Tomography is 
recommended. ${ }^{14}$ Preoperative ultrasonography of abdomen of our case showed feature of abdominal obstruction. Location of the tumour was in the proximal sigmoid colon. Rao et al showed that in children these neoplasms occurred in the cecum, ascending and descending colon, in descending order of frequency. ${ }^{17}$ Histopathological report of our patient showed that it was a moderately differentiated adenocarcinoma with lymph node metastases. Many other studies also showed that colorectal carcinomas were advanced stage at the time of diagnosis. ${ }^{6,18,19}$ Johnson et al found the incidence of poorly differentiated lesion with lymph node involvement is higher in children where as Middel and Haffner found $48 \%$ colon cancer in children were mucin secreting adenocarcinoma with signet ring formation. ${ }^{12,} 20$

Pre-operative CEA level in our case was not done but the CEA level after 8 weeks of operation was normal. In case of pediatric patient the prognostic value of CEA is undetermined, although increased preoperative CEA levels may be correlated with a higher rate of relapse. ${ }^{15,} 21,22$ Hemicolectomy was done in our patient, although the treatment of choice for the colonic carcinoma is complete resection to improve the survival time and rates. ${ }^{17,23,24}$

In large-group follow-up studies, the 5-year survival rate was worse in adolescents than in older patients (51\% vs. $75 \%) .{ }^{14}, 15$ When colorectal carcinoma is diagnosed and treated at Duke's stage A or B, a cure is possible but majority of childhood colorectal carcinoma are diagnosed late at Duke's stages $C$ or D, with a poor 5-year survival of $10 \%$ to $20 \%{ }^{14,15}$

\section{Conclusion}

Early detection and appropriate treatment increase the chance of cure of carcinoma colon in children. Children with recurrent abdominal pain of unknown etiology should be carefully evaluated. Suspicion should be kept in mind about the occurrence of colon carcinoma in children and mandatory diagnostic modalities such as abdominal ultrasound, barium studies, and/or colonoscopy should be carefully performed in children presenting with persistent abdominal pain of unknown etiology.

\section{Acknowledgement}

Prof. Mohammed Golam Mostofa, Head of the department of Histopathology, National Institute of Cancer Research and Hospital, Mohakhali, Dhaka 1212.

\section{References}

1. Pratt CB, Rivera G, Shanks E. Coloreetal carcinoma in adolescents: implications regarding aetiology. Cancer 1977; 40:2472.

2. Ries LA, Wingo PA, Miller DS, Howe HL, Weir $\mathrm{HK}$, Rosenberg HM et al. The annual report to the nation on the status of cancer, 1973-1997, with a special section on colorectal cancer. Cancer 2000; 88; 2398-2424.

3. Saab R, Furman WL. Epidemiology and management options for colorectal cancer in children. Paediatr Drugs 2008; 10; 177-192.

4. Colorectal cancer incidence and mortality worldwide in 2008. Summary. GLOBOCAN 2008.

5. Kern WH, White WC. Adenocarcinoma of the colon in a 9-month-old infant: report of a case. Cancer 1958; 11:855-857.

6. Hill DA, Furman WL, Billups CA, Riedly SE, Cain $\mathrm{AM}, \mathrm{Rao} \mathrm{BN}$ et al. Colorectal carcinoma in childhood and adolescent: a clinicopathologic review. J Clin oncol 2007:25:5808-5814.

7. Lewis CTP, Riley K, Georgeson HK. Carcinoma of the colon and rectum in patients less than 20 years of age. South Afr Med 1990; 83:383-385.

8. Tang T, Wang JY, Lo SK. Hsieh. Physical activity, water intake and risk of colorectal cancer in Taiwan: a hospital-based case control study. Int J Cancer 1999; 82:488

9. Culter SJ. Proceedings: report on the Third National Cancer Survey. Proc Natl Cancer Conf 1973; 7:639-52.

10. The Committee on Malignant Tumors, The Japanese Society of Pediatric Surgeons: Japanese Tumor Registry of Pediatric Tumors in 1984. Jpn Pediatr Surg 1986; 22:125-71.

11. Hsiang-Hung Shih, Chung-Ching Lu, Mao-Meng Tiao, Sheung-Fat Ko, Jiin-Haur Chuang. Adenocarcinoma of the Colon in Children Presenting as Abdominal Pain: Report of Two Cases. Chang Gung Med J 2002; 25:349-54.

12. Middelkamp JN, Haffner H. Carcinoma of the colon in children. Pediatrics 1963; 32:558-71.

13. Taguchi T, Suita S, Hirata Y. Carcinoma of the colon in children: a case report and review of 41 
Japanese cases. J Pediatr Gastroenterol Nutr 1991; 12:394-9.

14. Brown RA, Rode H, Millar AJW. Colorectal carcinoma in children. J Pediatr Surg 1992; 27:919-21.

15. Gold-thorn JF, Powars D, Hays DM. Adenocarcinoma of colon and rectum in the adolescent. Surgery 1983; 93:409-414.

16. Doraiswamy NV, Ahmed J. Carcinoma of the rectum in preteen girl. Pediatr Surg Int 1987; 2:124-6.

17. Rao NB, Pratt BC, Fleming DI. Colon carcinoma in children and adolescents: a review of 30 cases. Cancer 1985; 55:1322-6.

18. Radhakrishnan CN, Bruce J. Colorectal Cancer in children without any predisposing factors: A report of eight cases and review of the literature. Eur J Pediatr Surg 2003; 13:66-68.

19. Sharma AK, Gupta CR. Colorectal cancer in children: case report and review of literature. Trop Gastroenterol 2001. 22; 36-39.
20. Johnson JW, Judd ES, Dahlin DC. Malignant neoplasms of the colon and rectum in the young persons. Arch Surg 1959; 79:365-372.

21. Wanebo HJ, Rao BN, Pinsky CM. Preoperative carcinoembryonic antigen level as a prognostic indicator in colorectal cancer. N Engl J Med 1978; 299:448-51.

22. Wolmark N, Fisher B, Wieand S. Prognostic significance of preoperative carcinoembryonic antigen levels in colorectal cancer: Results from the NSABP clinical trials. Ann Surg 1984; 199:375-81.

23. Singer G, Hoellwarth ME. Colorectal carcinomas in children: an institutional experience. Pediatr Surg Int. 2012 Jun; 28:591-5.

24. Ferrari A, Rognone A, Casanova M, Zaffignani E, Piva L, Collini P et al. Colorectal carcinoma in children and adolescents: the experience of the Istituto Nazionale Tumori of Milan, Italy. Pediatr Blood Cancer. 2008 Mar; 50:588-93. 